\title{
Association between resting-state functional connectivity of amygdala subregions and peripheral pro-inflammation cytokines levels in bipolar disorder
}

\section{Jiaying Gong}

Sun Yat-sen University Sixth Affiliated Hospital https://orcid.org/0000-0002-8690-4451

\section{Guanmao Chen}

Jinan University First Affiliated Hospital

\section{Feng Chen}

Jinan University First Affiliated Hospital

\section{Shuming Zhong}

Jinan University First Affiliated Hospital

\section{Pan Chen}

Jinan University First Affiliated Hospital

Hui Zhong Jinan University

\section{Shunkai Lai}

Jinan University First Affiliated Hospital

\section{Guixian Tang}

Jinan University First Affiliated Hospital

Jurong Wang

Jinan University First Affiliated Hospital

\section{Zhenye Luo}

Jinan University First Affiliated Hospital

\section{Zhangzhang Qi}

Jinan University First Affiliated Hospital

\section{Yanbin Jia}

Jinan University First Affiliated Hospital

\section{Li Huang}

Jinan University First Affiliated Hospital

Ying Wang ( $\sim$ johneil@vip.sina.com )

Jinan University First Affiliated Hospital https://orcid.org/0000-0002-0388-4177 
Research

Keywords: Bipolar disorder, Inflammation, Functional magnetic resonance imaging, Functional connectivity, Amygdala.

Posted Date: October 2nd, 2020

DOI: https://doi.org/10.21203/rs.3.rs-85338/v1

License: (c) (i) This work is licensed under a Creative Commons Attribution 4.0 International License.

Read Full License

Version of Record: A version of this preprint was published at Brain Imaging and Behavior on February 17th, 2022. See the published version at https://doi.org/10.1007/s11682-022-00636-7. 


\section{Abstract}

Background: The pathophysiological mechanisms of mood disorders including bipolar disorder (BD) are not completely known, and systemic inflammation and immune dysregulation are considered as risk factors of it. Previous neuroimaging studies have proved metabolic, structural and functional abnormalities of the amygdala in BD patients, suggesting the vital role of amygdala in BD patients. This study aimed to test the underlying neural mechanism of inflammation-induced functional connectivity $(\mathrm{FC})$ in the amygdala subregions of BD patients.

Methods: Resting-state functional MRI (rs-fMRI) was used to delineate the amygdala FC from two pairs of amygdala seed regions (the bilateral lateral and medial amygdala) in 51 unmedicated BD patients and 69 healthy controls (HCs). The levels of pro-inflammatory cytokines including interleukin (IL)-1 $\beta$, IL-6 and tumor necrosis factor (TNF)-a were measured in the serum. The correlation between abnormal levels of pro-inflammatory cytokines and FC values were calculated in BD patients.

Results: The BD group exhibited decreased FC between the right medial amygdala and bilateral medial frontal cortex (MFC), and decreased FC between the left medial amygdala and the left temporal pole (TP), right orbital inferior frontal gyrus compared with HCs. The BD patients had higher levels of TNF- $a$ than HCs. Correlation analysis showed negative correlation between the TNF-a level and abnormal FC of the right medial amygdala-bilateral MFC; and negative correlation between TNF-a levels and abnormal FC of the left medial amygdala-left TP in BD group.

Conclusions: These findings suggest that dysfunctional and immune dysregulation between the amygdala and the frontotemporal circuitry might play a critical role in the pathogenesis of BD.

\section{Background}

Bipolar disorder (BD) is a chronic, recurrent and disabling psychiatric illness that causes cognitive impairment and dysfunction and reduces quality of life, and is associated with high morbidity and mortality $[1,2]$. Early and accurate diagnosis of BD is difficult in clinical practice, and it is often misdiagnosed as unipolar depression as the onset of BD is commonly characterized by nonspecific symptoms, mood lability or a depressive episode. Although it has been studied for over decades, the pathophysiology of BD remained unclear. Dysfunction of innate immune system that leads to neuroinflammation has been considered as risk factor in the pathophysiology of numerous psychiatric illnesses including BD [3-6].

Resting-state functional magnetic resonance imaging (rs-fMRI) is a primary noninvasive neuroimaging technique that can reflect the changes of brain function status and resting state functional connectivity (rs-FC), and is a fundamental tool in reflecting the correlations of activities between different brain regions, helping to understand brain regions with altered connectivity and function in mental disorders [7, 8]. Previous neuroimaging studies have reported that the amygdala abnormity, including reduced gray matter volume [9], broken white matter integrity, decreased FC [10] and lower activation [11], ais 
associated with the pathophysiology of BD. The amygdala is a complex consisting of structural and functional heterogeneous nuclei, tucked in the medial temporal lobe and comparatively small in size. It plays a central role in regulating emotions and mediating fear responses [12,13]. The amygdala complex is grouped into three distinct subregions cytoarchitectonically: centromedial, basolateral, and superficial $[12,14]$. As there several studies that discussed different functions of centromedial (including the central and medial nuclei) and basolateral (including the lateral, basolateral, basomedial, and basoventral nuclei) amygdala, but not the superficial region [14-16], this study focused on the centromedial and basolateral subregions that are similar to the medial and lateral masks according to the Brainnetome atlas [14]. The lateral amygdala receives sensory information and involves assessment of emotional content of sensory information, while the medial amygdala is regarded as the major output station of the entire amygdala and involves enhancement of attention allocation and determine the salience of input $[13,17,18]$. Existing studies have put forwarded the changes in amygdala FC that are related to BD accompanied by the frontal lobe (including the dorsolateral prefrontal cortex [19], prefrontal cortex [20], and inferior frontal gyrus [IFG] [21]), supplementary motor area [22] and anterior hippocampus [23]. Among these studies, only one study used the amygdala subregions FC, but through region of interest (ROI)-to-ROI analyses instead of whole-brain approach based on subregions seed masks of each subregion [23]. However, the functional connectivity between the amygdala subregions and the brain areas has not been examined in detailed manner in BD.

Cytokines act as regulators of host responses to infection, inflammation, trauma, and immune responses [24]. During the process of inflammation, proinflammatory cytokines access the central nervous system and interact with cytokine network in the brain to virtually influence each and every aspect of brain function that is relevant to the behavior including neurotransmitter metabolism, neuroendocrine function, synaptic plasticity, mood regulation, motor activity, motivation, anxiety and panic [25]. Immune system dysfunction leads to the occurrence of neuroinflammation in BD and increasingly implicated in the pathophysiology of this mental disorder [26, 27]. Several studies have shown elevated proinflammatory cytokines including but not limited to IL-6, IL-1 $\beta$, and TNF- $\alpha$ in patients with BD $[4,28]$, particular in acute mania [29-32] and depressive episodes [29-32]. Previous meta-analyses have reported that IL-1 3 , IL-6, and TNF- $a$ are the most common abnormal cytokines in BD patients $[3,29,33]$. Some MRI studies have revealed abnormal structure and dysfunction in specific brain regions (such as amygdala, IFG, medial frontal cortex [MFC], and temporal lobe [TP]) and its correlation with IL-1 $\beta$, IL-6, and TNF- $\alpha$ in the peripheral area, and might contribute to the symptoms of fear, anxiety and emotional disturbances. This might help us to better understand the role of IL-1 $\beta, I L-6$, and TNF- $\alpha$, and its relation to the pathophysiology of mood disorder [32, 34-36]. A rs-fMRI study revealed that higher IL-6 receptor levels are associated with connectivity abnormalities between the medial prefrontal cortex and amygdala involved in BD [37].

In our study, the whole-brain FC of each amygdala subdivision in BD patients BD and HCs was calculated, and the levels of IL-1 $\beta, I L-6$, and TNF-a were obtained in the peripheral blood of these participants. This study aimed to explore the neural mechanism of inflammation induced in the amygdala subdivisions FC 
in patients with $\mathrm{BD}$. We hypothesized that both the amygdala subdivisions $\mathrm{FC}$ and pro-inflammatory cytokines levels are abnormal, and the amygdala dysfunction is linked to the changes in cytokines.

\section{Materials And Methods}

\section{Participants}

A total of 55 right-handed individuals diagnosed with BD (15 BD $\otimes$ and $40 \mathrm{BD} \otimes)$ were recruited from the in- and out-patients of psychiatry department, First Affiliated Hospital of Jinan University, Guangzhou, China. The patients' age ranged from 18 to 55 years. All patients met the Diagnostic and Statistical Manual of Mental Disorders, Fifth Edition (known as DSM-V) criteria for BD according to the diagnostic assessment by the Structured Clinical Interview for DSM-V Patient Edition (SCID-P) put forwarded by two experienced psychiatrists (Y.J. and S.Z., with 21 and 6 years of experienced clinical psychiatry, respectively). The clinical state of $\mathrm{BD}$ patients was assessed by using the 24-item Hamilton Depression Rating Scale (HDRS) and the Young Mania Rating Scale (YMRS) during the 3-day period prior to the imaging session. The exclusion criteria included patients with other Axis-I psychiatric disorders as assessed by two experienced psychiatrists (Y.J. and S.Z., with 21 and 6 years of experienced clinical psychiatry, respectively) through SCID-P, a history of electroconvulsive therapy, other neurological disorders, any history of organic brain disorder, autoimmune/immune diseases, mental retardation, pregnancy, alcohol/substance abuse, cardiovascular diseases or presence of any concurrent and major physical illnesses as reported by laboratory examination or self-reported by patients in the experienced psychiatrists' interview and when conversing with patients. Those patients who currently took antiinflammatory medications or immunomodulators (i.e., statins or metformin) were excluded from the study. At the time of testing, all patients were either medication-naive, or were not medicated for at least six months. In addition, 70 right-handed healthy controls (HCs) were recruited via local advertisements. They were carefully screened through a diagnostic interview, the Structured Clinical Interview for DSM-V Nonpatient Edition (SCID-NP), to rule out the presence of current or past history of psychiatric illnesses. Further exclusion criteria for HCs include history of psychiatric illnesses in first-degree relatives, current or past significant medical or neurological illnesses.

This study was approved by the Ethics Committee of First Affiliated Hospital of Jinan University, Guangzhou, China. All subjects were right-handed and signed a written informed consent form after full writing and verbal explanation of the study. Two senior clinical psychiatrists have confirmed that all subjects had the ability to participate in the examination.

\section{MR imaging data acquisition and preprocessing}

All MRI data were obtained by using a GE Discovery MR 750 3.0T System with an 8-channel phased array head coil. Subjects were scanned in a supine, head-first position with symmetrically placed cushions on both sides of the head to decrease motion. During the scanning, participants were instructed to relax with their eyes closed without falling asleep; and after the experiment, each participant confirmed not having fallen asleep. 
The rs-fMRI data were acquired using gradient-echo echo-planar imaging sequence with the following parameters: time repetition $(T R) /$ time echo $(T E)=2000 / 25 \mathrm{~ms}$, flip angle $=90^{\circ}$, voxel size $=3.75 \times 3.75 \times 3$ $\mathrm{mm}^{3}$, field of view $(F O V)=240 \times 240 \mathrm{~mm}$, matrix $=64 \times 64$, slice thickness $/ \mathrm{gap}=3.0 / 1.0 \mathrm{~mm}, 35$ axial slices covering the whole-brain, and 210 volumes acquired in 7 minutes. In addition, a three dimensional brain volume imaging (3D-BRAVO) sequence covering the whole brain was used for structural data acquisition with: $\mathrm{TR} / \mathrm{TE}=8.2 / 3.2 \mathrm{~ms}$, flip angle $=12^{\circ}$, bandwidth $=31.25 \mathrm{~Hz}$, slice thickness $/ \mathrm{gap}=$ $1.0 / 0 \mathrm{~mm}$, matrix $=256 \times 256, \mathrm{FOV}=240 \times 240 \mathrm{~mm}, \mathrm{NEX}=1$, and acquisition time $=3 \mathrm{~min} 45 \mathrm{~s}$. Routine MRI examination images were also collected for excluding anatomic abnormality. All participants were found by two experienced neuroradiologists to confirm the absence of any brain structural abnormalities.

\section{Functional Image Preprocessing}

The preprocessing was carried out using the Data Processing Assistant for Resting-State fMRI (DPABI_V3.0, http://restfmri.net/forum/DPABI) [38], and this is based on Statistical Parametric Mapping (SPM12, http://www.fil.ion.ucl.ac.uk/spm/). For each subject, the first 10 images of the rs-fMRI dataset were discarded to ensure steady-state longitudinal magnetization. The remaining 200 images were slicetime corrected initially and then were realigned to the first image for correcting the inter-TR head motion. This realignment correction provides a record of the head motion within the rs-fMRI scan. All subjects should not have more than $2 \mathrm{~mm}$ maximum displacement in any plane, $2^{\circ}$ of angular motion as well as $0.2 \mathrm{~mm}$ in the mean frame-wise displacement (FD) [39]. The individual T1 structural images were segmented (white matter, gray matter, and cerebrospinal fluid) using a segmentation toolbox. The DARTEL toolbox was then used to create a study specific template for accurate normalization. The resting-state functional images were co-registered with the structural images and transformed into the standard Montreal Neurological Institute (MNI) space, resliced to a voxel size of $3 \times 3 \times 3 \mathrm{~mm}^{3}$ resolution and smoothened using a $6 \mathrm{~mm}$ full width at half maximum (FWHM) Gaussian kernel. The data were removed the linear trend and passed through the band-pass filter of $0.01-0.1 \mathrm{~Hz}$. The global mean signal regression is commonly used in the field of rs-fMRI, and it could afford decreased dependencies on head motion [40] and increased tissue sensitivity [41]. The signals from white matter and cerebrospinal fluid were regressed out to reduce both respiratory and cardiac effects [42]. The whole brain functional connectivity estimates were influenced by head motion and the head motion could be misinterpreted as neuronal effects easily [43]. Several spurious covariates and their temporal derivatives were then regressed out from the time course of each voxel, including the signals of the brain global mean, white matter, and cerebrospinal fluid as well as the Friston-24 parameters of head motion (including the 6 head motion parameters, 6 head motion parameters at one time point before, and the 12 corresponding squared items) [44].

\section{Resting-state Functional Connectivity}

Consistent with the previous studies of amygdala FC [14], four seed masks (the bilateral lateral amygdala and medial amygdala) were selected using Brainnetome atlas (http://atlas.brainnetome.org/bnatlas.php) [45]. The time course of the masks was correlated against all other voxels within the whole brain. 
Individual rs-FC maps of the lateral amygdala, and medial amygdala were generated by calculating the Pearson's correlation coefficients between the mean time series of the masks and the time series of each voxel in the whole brain. The subject-level correlation maps were then converted to the z-value maps using Fisher's transformation to improve the normality. For all the subjects, four z-score maps that represent the intrinsic FC of the four amygdala masks were finally obtained.

\section{Pro-inflammatory Cytokines Measures}

Blood samples from BD patients and HCs were obtained in the morning under fasting condition, followed by abstaining by alcoholic beverages for at least one day prior to testing and then were processed (then frozen) by the technicians. Through venipuncture, four milliliters of fasting blood were drawn from each subject into a sterile vacuum tube (Becton \& Dickinson 367812; Becton Dickinson, Franklin Lakes, NJ, USA), clotted for $30 \mathrm{~min}$, and then centrifuged at $1,000 \mathrm{~g}$ for $15 \mathrm{~min}$ at $4{ }^{\circ} \mathrm{C}$. The serum was centrifuged again $\left(3,000 \mathrm{rpm}\right.$ for $10 \mathrm{~min}$ at $\left.4{ }^{\circ} \mathrm{C}\right)$, and stored at $-80^{\circ} \mathrm{C}$ until use. Before testing, the serum should be melted on ice at low temperature, and the testing process was carried out at room temperature in accordance with the requirements of the kit to ensure effective combining of antigen and antibody. The levels of pro-inflammatory cytokines, including IL-6, IL-1 $\beta$, and TNF-a were determined from the serum by the Bio-Plex Pro Human Cytokine Assay kit (Bio-Rad) according to the manufacturer's directions using a Bio-Plex 200 array reader (Bio-Rad). Bio-Plex Manager Software, version 6.1 was used for data acquisition (Bio-Rad). The intra-assay coefficients of variation (CVs) were $3.58 \%$ for IL-1 $3,2.97 \%$ for IL-6, and $3.66 \%$ for TNF-a. A reliable detection range was provided with the lower limit of quantitation (LLOQ) and the upper limit of quantitation (ULOQ). The detection ranges (LLOQ-ULOQ) were 0.057$1027.514 \mathrm{pg} / \mathrm{ml}$ for IL-1 $\beta, 0.11-1997.01 \mathrm{pg} / \mathrm{ml}$ for IL-6, and 0.69-13336.72 pg/ml for TNF-a. No immune variables were below the limits of assay detection.

\section{Statistical analysis}

Independent-sample t-test (normal variable) and Mann-Whitney $U$ test (non-normal variable) were used to compare the demographic data (except gender) and levels of serum pro-inflammatory cytokines between the two groups with SPSS 19.0 software (SPSS, Chicago, IL, USA). A chi-squared test was performed to compare gender distribution. All tests were two-tailed, and $p<0.05$ was considered to be statistically significant. One-sample t-test was performed on z-score maps for each mask to demonstrate withingroup FC spatial distribution of each seed for BD patients and HCs, and the significant level was set at $p$ $<0.05$ (uncorrected). Then the two-sample $t$-test was performed to assess significant differences of the whole brain FC in each region between BD patients and HCs within the union mask of one-sample $t$-test results of both groups. Age, gender, years of education and the mean FD were included as nuisance covariates in the whole brain FC and cytokine group comparisons. Gaussian random field (GRF) theory was used for cluster-level multiple comparison correction (voxel $p$ value $<0.001$; cluster $p$ value $<0.05$, GRF corrected). When statistically significant group differences were observed in the brain regions and pro-inflammatory cytokines levels, Spearman correlation analysis was performed to compute the correlation between FC values and pro-inflammatory cytokine levels both in BD and HC groups. Also, 
Spearman correlation coefficients were calculated between the clinical variables and abnormal FC values, and abnormal pro-inflammatory cytokines levels in the BD group. These clinical variables included onset age of illness, number of episodes, duration of illness, 24-item HDRS scores and YMRS scores. Further, multiple linear regression was adopted to model the relationship between FC values and proinflammatory cytokines levels after adjusting for potential confounders including demographic variables, number of cigarettes, body mass index (BMI), and BD subgroup variable ("1" for BD I and "2" for BD II). The significant level was set as $p<0.05$.

\section{Results}

\section{Demographic and clinical characteristics}

The demographic and clinical data of all study participants were presented in Table 1. Four patients in BD (three BD I and one BD II) group and one $\mathrm{HC}$ were excluded from further analyses because of excessive head motion. Finally, 51 patients with $\mathrm{BD}(12 \mathrm{BD} \otimes$ and $39 \mathrm{BD} \otimes)$ and $69 \mathrm{HCs}$ were included in the analysis. The 24-item HDRS score was 23.14 (9.94), and the YMRS score was 8.57 (9.13) for the BD group. There were no significant differences between the BD group and the HCs group in gender, age, BMI, or FD parameters $(p>0.05)$. 
Table 1

Demographic and clinical data and (standard deviations) by group

\section{Number of subjects}

Bipolar disorderControl $p$ value

\begin{tabular}{llll} 
Age (years) & $27.84(8.63)$ & $31.48(11.62)$ & 0.052 \\
\hline Gender (male/female) & $24 / 27$ & $33 / 36$ & $0.978+$ \\
\hline BMI (kg/m2) & $21.43(3.62)$ & $20.44(2.24)$ & 0.274 \\
\hline Smoking (cigarettes/day) & $0.98(3.67)$ & - & \\
\hline Age at onset (years) & $24.0(8.60)$ & $\mathrm{n} / \mathrm{a}$ & \\
\hline Number of episodes & $2.92(1.60)$ & $\mathrm{n} / \mathrm{a}$ & \\
\hline 24-item HDRS score & $23.14(9.94)$ & $\mathrm{n} / \mathrm{a}$ & \\
\hline YMRS score & $8.57(9.13)$ & $\mathrm{n} / \mathrm{a}$ & 0.415 \\
\hline Duration of illness (months) & $39.21(49.96)$ & $\mathrm{n} / \mathrm{a}$ & 0.166 \\
\hline FD & $0.046(0.028)$ & $0.05(0.027)$ & 0.054 \\
\hline IL-1 $\beta$ & $0.79(0.73,0.86)^{\#}$ & $0.79(0.73,0.86)$ & $0.034^{*}$ \\
\hline IL-6 & $2.57(2.18,2.83)^{\#}$ & $2.31(2.05,2.57)$ & \\
\hline TNF-a & $20.90(16.73,25.01)^{\#}$ & $18.13(16.73,21.24)$ & \\
\hline
\end{tabular}

Means (with standard deviations in parentheses) are reported unless otherwise noted.

*The $p$ values were obtained by Mann-Whitney $U$ tests.

tThe $p$ value for gender distribution was obtained by chi-square test.

\# The mean is denoted by the median and the quartile.

BMI, Body Mass Index; HDRS, Hamilton Depression Rating Scale; YMRS, Young Mania Rating Scale; FD, framewise displacement for in-scanner head motion; IL, interleukin; TNF, tumor necrosis factor.

\section{FC analysis within-group}

One-sample $t$-test displayed the within-group FC patterns in the BD and HCs groups. The one-sample $t$ test revealed that the bilateral lateral amygdala subregions with high $\mathrm{FC}$ values were mainly connected to the cerebellum, parahippocampal gyrus, insula, putamen, superior/middle/inferior temporal gyrus, TP, superior/middle/inferior frontal gyrus, cingulate gyrus, pre/postcentral gyrus, supplementary motor area and parietal lobe in the HCs group. The bilateral medial amygdala subregions with high FC values were 
mainly connected to the parahippocampal gyrus, inferior/middle/superior temporal gyrus, insula, putamen, parietal lobe, cingulate gyrus, MFC, IFG, TP, pre/postcentral gyrus, supplementary motor area, precuneus, cingulate gyrus and cerebellum in the HCs group. The FC spatial distribution in the BD group was similar to that of the HCs group with visual inspection (Fig. 1).

\section{FC changes between-group}

Decreased FC between the right medial amygdala and the bilateral MFC and decreased FC between the left medial amygdala and the left TP, the right orbital IFG were observed in the BD group when compared to the $\mathrm{HC}$ group. There was no aberrant FC between the right/left lateral amygdala and the cerebral regions in the BD group was observed when compared to the HC group (Table 2 and Fig. 2).

Table 2

The areas of significantly different FC between the BD patients and the HCs (GRF correction, voxel $p<$ 0.001 , cluster $p<0.05)$.

\begin{tabular}{|c|c|c|c|c|c|c|c|}
\hline \multirow[t]{2}{*}{ Seeds } & \multirow[t]{2}{*}{$\begin{array}{l}\text { location in } \\
\text { the } \\
\text { cerebrum }\end{array}$} & \multirow[t]{2}{*}{$\begin{array}{l}\text { Brodman } \\
\text { Area }\end{array}$} & \multicolumn{3}{|c|}{$\begin{array}{l}\text { Montreal } \\
\text { Neurological } \\
\text { Institute } \\
\text { Coordinates }\end{array}$} & \multirow[t]{2}{*}{$\begin{array}{l}\text { Peak } t \\
\text { Value }\end{array}$} & \multirow[t]{2}{*}{$\begin{array}{l}\text { Cluster Size } \\
\text { (voxel } \\
\text { numbers) }\end{array}$} \\
\hline & & & $x$ & $Y$ & Z & & \\
\hline \multirow{2}{*}{$\begin{array}{l}\text { L medial } \\
\text { amygdala }\end{array}$} & L TP & 20 & -42 & 15 & -27 & -4.503 & 45 \\
\hline & $\begin{array}{l}\text { R orbital } \\
\text { IFG }\end{array}$ & 38 & 45 & 27 & -15 & -4.282 & 39 \\
\hline $\begin{array}{l}\text { R medial } \\
\text { amygdala }\end{array}$ & $\begin{array}{l}\text { Bilateral } \\
\text { MFC }\end{array}$ & 11 & 3 & 48 & -18 & -4.435 & 56 \\
\hline
\end{tabular}

\section{Group Differences Of Pro-inflammatory Cytokines Levels}

The levels of IL-6, IL-1 $\beta$, and TNF- $a$ of the two groups were shown in Table 1 . The measurements of serum pro-inflammatory cytokines levels were completed in $51 \mathrm{BD}$ patients and $69 \mathrm{HCs}$. No significant differences were observed in sex, age, and FD parameter between the two groups. Compared with $\mathrm{HCs}$, BD patients showed increased TNF-a levels $(p<0.05)$. No statistical differences of IL- 6 and IL-1 $\beta$ were observed between the two groups $(p>0.05)$ (Table 1).

\section{Correlation Analyses}

For BD patients, the FC values in the right medial amygdala-bilateral MFC showed a negative correlation with TNF-a $(r=-0.314, p=0.025)$ level; and the FC values in the left medial amygdala-left TP showed a 
negative correlation with TNF-a $(r=-0.330, p=0.018)$ level (Fig. 3). For HC group, no significant correlations between the FC values in the right medial amygdala-bilateral MFC and TNF-a $(r=0.048, p=$ 0.696), and between the FC values in the left medial amygdala-left TP and TNF- $a(r<0.001, p=0.997)$ were observed.

For the correlations between FC values and clinical variables, including onset age of illness, number of episodes, duration of illness, 24-item HDRS score, and YMRS score, and between levels of TNF-a and clinical variables in BD patients, no significant correlation of survival was observed when Spearman correlation analysis was performed $(p>0.05)$.

After adjusting for demographic variables, number of cigarettes, BMI, and BD subgroup variable in BD group, the TNF-a showed a negative correlation with the FC values in the right medial amygdala-bilateral MFC (beta $=-0.302, t=-2.184, p=0.032$ ), and a negative association with the FC values in the left medial amygdala-left TP (beta $=-0.395, t=-2.694, p=0.011$ ). Age, number of cigarettes, $\mathrm{BMI}$, and BD subgroup variable showed no association with TNF- $a$, the FC values in the right medial amygdala-bilateral MFC, and the FC values in the left medial amygdala-left TP (all $p>0.05$ ). In addition, there were no significant relationships between FC values and pro-inflammatory cytokines levels in the HC group.

\section{Discussion}

We performed whole brain amygdala subregions rs-FC and measured the levels of pro-inflammatory cytokines including IL- $6, \mathrm{IL}-1 \beta$, and TNF- $\alpha$ in the serum of BD patients and HCs. The primary findings of this study were as follows: (i). decreased FC was observed between the right medial amygdala and the bilateral MFC, and decreased FC between the left medial amygdala and the left TP, the right orbital IFG in BD group; (ii). increased TNF-a levels were observed in BD patients than that in HCs; (iii). negative correlation was observed between the TNF-a levels and abnormal FC in the right medial amygdalabilateral MFC; and negative correlation between the TNF-a levels and abnormal FC in the left medial amygdala-left TP was observed in BD patients. To the best of our knowledge, this is the first study to assess the association between the amygdala subregion rs-FC and inflammatory cytokines levels in BD patients, and this assists us to raise awareness with regard to BD from the perspective of intrinsic brain activity and immune system.

In the present study, patients with BD showed decreased FC between the right medial amygdala and the bilateral MFC, suggesting a disturbed connectivity between the amygdala and MFC in BD patients. The MFC has been assumed to play a role in emotional regulation by generating preventive responses preceding the emotional events [46]. Previous rs-fMRI studies have shown decreased FC of the MFC and IFG in patients with $\mathrm{BD}$, and this has shed light on the underlying pathophysiology and presentation of impaired emotion regulation and cognitive control [47-50]. Furthermore, another rs-fMRI study using voxel-mirrored homotopic connectivity (VMHC) has investigated that lower VMHC in the MFC in BD patients. VMHC, is a common rs-fMRI method, that measures the functional connection between each voxel in one hemisphere and the mirror voxel in the other hemisphere, suggesting substantial impairment 
of interhemispheric coordination in BD patients [51]. Study of regional function in BD patients showed decreased activity in the MFC [52] and IFG regions [53]. Recent task-based fMRI investigation found that patients with BD had neural dysfunction in the MFC, implicating appraisal and expression of emotions [54]. Vai et al have reported that successful antidepressant chronotherapeutics can enhance fronto-limbic connectivity (MFC-amygdala) in emotional processing neural responses in BD patients [55]. Postmortem studies have revealed dendritic atrophy, oligodendrocyte loss, and gray matter loss in the MFC in BD patients, raising the possibility of abnormal synaptic interactions between the MPFC, amygdala, and OFC among other regions. This may contribute to the disturbances in emotional regulation characteristics of $\mathrm{BD}[56,57]$. Taken together, our findings of decreased FC between the right medial amygdala and the bilateral MFC might show an abnormal brain integration in default processing of emotional information, displaying the clinical sign of emotional dysregulation in patients with BD.

The TP covering the anterior-most part of the temporal lobe, like a cap lies between the orbital frontal cortex and the amygdala, receives and sends the connections to both regions, playing an important role in socioemotional processing [58]. In our study, decreased FC was found between the left medial amygdala and the left TP, suggesting disrupted connectivity between the amygdala and the TP. Another fMRI study of BD also exhibited decreased FC from the amygdala to the TP, and this was consistent with our results [59]. Other several rs-fMRI studies have shown decreased FC of the TP in participants with psychiatric symptoms [60-62]. Other studies have shown TP abnormalities, including decreased gray matter density [63], reduced gray matter volume [64, 65], lower mean diffusivity (reflects the microstructural integrity of white matter) [66], and decreased degree centrality (reflects the functional connections)[67] in BD patients. This suggested that the abnormal structure and connectivity alterations might underlie the mood fluctuations and cognitive and emotional dysregulation that characterize BD. One task-based fMRI research on viewing affective or neutral faces tasks showed that both euthymic and manic BD patients have displayed reduced activation than HCs in the TP [68]. A magnetoencephalography study investigated implicit emotional tasks that showed abnormal activation in the temporal cortices in BD [69]. Thus, our findings of deficient FC between the amygdala and the TP might be related to emotional dysregulation of $\mathrm{BD}$, explaining the neurobiological processes that underlie impaired emotion processing in BD.

Cytokines play a significant role in stimulating and maintaining the immune processes, and strong evidence has indicated that TNF-a could mediate the clinical presentation of BD and its neurobiological manifestations [70]. TNF-a has been described as a major inflammatory mediator, that promote the production of other cytokines such as IL-6 and C-reactive protein [71]. In this study, increased TNF-a levels were found in BD patients when compared to those in $\mathrm{HCs}$, and this was consistent with most of the previous studies [3,72]. Moreover, a negative correlation was found between TNF-a levels and abnormal FC of the right medial amygdala-right MFC, and abnormal FC of the left medial amygdala-left TP in BD patients. Chronic inflammatory responses in patients with mood disorders can lead to downstream biological damage, causing damage to BD patients through upcoming actions and effects the neurotransmitter and neuropeptide systems [73]. Karabulut et al have found that TNF-a levels were correlated with Montgomery-Asberg Depression Rating Scale scores and Clinical Global Impression Scale 
scores in BD patients, suggesting the progression at later stages of BD and possible association with gliosis and neuronal loss [74]. According to an animal study, chronic unpredictable mild stress exposure induced microglia activation and overexpression of cytokine TNF- $a$ within the MFC, and the effects were paralleled with neuronal structural changes. In contrast, chronic administration of antagonists has significantly ameliorated the dysregulation of neuronal structure and biochemical parameters within the MFC, and displayed depression-like behaviors [75]. The cytokine (TNF-a) has been shown to cross the rodent blood-brain barrier, rapidly inducing the correlated-sensitive genes within the brain, and altering the neuronal firing rates within the amygdala and other brain regions [76]. Recent literatures have found that inflammatory stimulation leads to changes in the amygdala activity and connectivity, and that the production of stress-induced inflammatory cytokines is related to the enhancement of amygdala reactivity $[77,78]$. A rs-fMRI study has found that the FC between striatum and prefrontal cortices showed negative association with serum TNF-a levels in major depressive disorder patients [79]. Another fMRI study investigated that elevated levels of depression and serum cytokine levels were associated with increased regional activity in the medial frontal lobe [80]. Evidence was obtained for distinct mechanisms by which the MFC function interacts with the inflammatory cytokine TNF-a, and this can partly explain the chronic inflammatory of impaired mood regulations in BD. This might in turn serve as a target for antiinflammatory treatment strategies to improve emotion deficits in patients with increased inflammation, including $B D[75,80]$. However, transient elevation of inflammation might be a part of adaptation to the disease. For patients with $\mathrm{BD}$, more research is warranted to determine if the higher TNF-a level and decreased FC of the amygdala-MFC, and -TP were associated with illness or pathogenesis to BD.

\section{Limitation}

There are several limitations in this study that should be acknowledged. Firstly, patients with BD in mixed subtypes (including $B D \otimes$ and $B D \otimes$ ) were examined, and it is still unclear as to whether the altered $F C$ and TNF-a is shared by all episodes of the disease or specific to some subtypes of BD. Secondly, this study lacked longitudinal data and our understanding with regard to the functional role of individual cytokines is also limited. Thirdly, the findings of TNF showed no survival after applying Bonferonni correction. Fourthly, in order to avoid excessive measurement costs, all cytokines samples were measured in one experiment, but this may not achieve high reliability and sensitivity of the retest. Fifthly, all smoking information of HCs, that affects the immune system was missed in this study. Finally, a larger sample size was needed to conclude the alterations of TNF-a levels in patients with BD. Also, a structural study is also warranted to confirm the results, of determining whether the changes of FC and TNF-a levels in BD patients has structural basis.

\section{Conclusions}

Our findings of decreased FC between the right medial amygdala-right MFC, and between the left medial amygdala-left TP might reflect the disrupted cortico-amygdala connectivity in patients with BD, which might show correlation with immune dysregulation in $\mathrm{BD}$ patients to some extent. These findings suggest 
the importance of dysfunctional and immune-dysregulated cortico-amygdala circuitry in the pathogenesis of $\mathrm{BD}$.

\section{Abbreviations}

BD: bipolar disorder; FC: functional connectivity; rs-fMRI: Resting-state functional MRI; HCs: healthy controls; IL: interleukin; TNF: tumor necrosis factor; MFC: medial frontal cortex; TP: temporal pole; rs-FC: resting state functional connectivity; IFG: inferior frontal gyrus; ROI: region of interest; DSM-V: Diagnostic and Statistical Manual of Mental Disorders, Fifth Edition; SCID-P: Structured Clinical Interview for DSM-V Patient Edition; HDRS: Hamilton Depression Rating Scale; YMRS: Young Mania Rating Scale; SCID-NP: Structured Clinical Interview for DSM-V Nonpatient Edition; TR: time repetition; TE: time echo; FOV: field of view; 3D-BRAVO: three dimensional brain volume imaging; FD: frame-wise displacement; MNI: Montreal Neurological Institute; FWHM: full width at half maximum; CVs: coefficients of variation; LLOQ: lower limit of quantitation; ULOQ: upper limit of quantitation; GRF: Gaussian random field; BMI: body mass index; VMHC: voxel-mirrored homotopic connectivity.

\section{Declarations}

\section{Acknowledgements}

We would like acknowledge all funding organizations and participants of this study.

\section{Author contributions}

Ying Wang design the study; Jiaying Gong, Ying Wang contribute to data sources and study selection; Jiaying Gong, Guanmao Chen, Feng Chen, Shuming Zhong, Pan Chen, Hui Zhong, Shunkai Lai, Guixian Tang, Jurong Wang, Zhenye Luo, Zhangzhang Qi, Yanbin Jia contribute to data acquisition; Guanmao Chen, Feng Chen contribute to data analysis; Jiaying Gong write the manuscript; Pan Chen, Feng Chen, Jiaying Gong, Guanmao Chen, Feng Chen, Hui Zhong, Li Huang, Ying Wang revise the manuscript. All authors contribute to and have approved the final manuscript. We thank all the authors of the in. cluded studies who responded to our requests for further information.

\section{Funding}

The study was supported by grants from the National Natural Science Foundation of China (81671670 and 81971597); Project in Basic Research and Applied Basic Research in General Colleges and Universities of Guangdong, China (018KZDXM009); Planned Science and Technology Project of Guangzhou, China (201905010003). The funding organizations play no further role in study design, data collection, analysis and interpretation and paper writing.

\section{Availability of data and materials}


The datasets during and/or analyzed during the current study will be made available from the corresponding author on reasonable request.

\section{Ethics approval and consent to participate}

This study was approved by the Ethics Committee of First Affiliated Hospital of Jinan University, Guangzhou, China. All subjects signed a written informed consent form after full writing and verbal explanation of the study.

\section{Consent for publication}

Not applicable.

\section{Competing interests}

The authors have declared that no competing interest exists.

\section{Author details}

${ }^{a}$ Medical Imaging Center, First Affiliated Hospital of Jinan University, Guangzhou 510630, China

${ }^{b}$ Department of Radiology, Six Affiliated Hospital of Sun Yat-sen University, Guangzhou 510655, China

${ }^{c}$ Institute of Molecular and Functional Imaging, Jinan University, Guangzhou, 510630, China

${ }^{d}$ Department of Psychiatry, First Affiliated Hospital of Jinan University, Guangzhou 510630, China

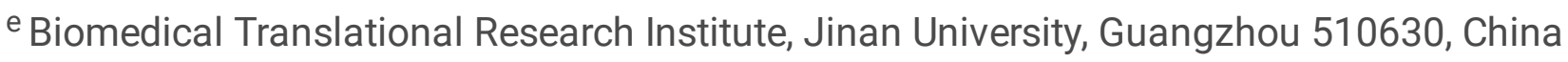

\section{References}

1. Kupfer DJ. The increasing medical burden in bipolar disorder. JAMA. 2005;293(20):2528-30.

2. Vieta E, Berk M, Schulze TG, Carvalho AF, Suppes T, Calabrese JR, et al. Bipolar disorders. Nat Rev Dis Primers. 2018;4:18008.

3. Goldsmith DR, Rapaport MH, Miller BJ. A meta-analysis of blood cytokine network alterations in psychiatric patients: comparisons between schizophrenia, bipolar disorder and depression. Mol Psychiatry. 2016;21(12):1696-709.

4. Brunoni AR, Supasitthumrong T, Teixeira AL, Vieira EL, Gattaz WF, Bensenor IM, et al. Differences in the immune-inflammatory profiles of unipolar and bipolar depression. J Affect Disord. 2020;262:815 .

5. Lin K, Shao R, Wang R, Lu W, Zou W, Chen K, et al. Inflammation, brain structure and cognition interrelations among individuals with differential risks for bipolar disorder. Brain Behav Immun. 2020;83:192-9. 
6. Dickerson F, Severance E, Yolken R. The microbiome, immunity, and schizophrenia and bipolar disorder. Brain Behav Immun. 2017;62:46-52.

7. Deco G, Kringelbach ML. Great expectations: using whole-brain computational connectomics for understanding neuropsychiatric disorders. Neuron. 2014;84(5):892-905.

8. Mulders PC, van Eijndhoven PF, Schene AH, Beckmann CF, Tendolkar I. Resting-state functional connectivity in major depressive disorder: A review. Neurosci Biobehav Rev. 2015;56:330-44.

9. Yu H, Meng YJ, Li XJ, Zhang C, Liang S, Li ML, et al. Common and distinct patterns of grey matter alterations in borderline personality disorder and bipolar disorder: voxel-based meta-analysis. $\mathrm{Br} \mathrm{J}$ Psychiatry. 2019;215(1):395-403.

10. Johnston J, Wang F, Liu J, Blond BN, Wallace A, Liu J, et al. Multimodal Neuroimaging of Frontolimbic Structure and Function Associated with Suicide Attempts in Adolescents and Young Adults with Bipolar Disorder. Am J Psychiatry. 2017;174(7):667-75.

11. Jesudas BR, Nandeesha H, Menon V, Allimuthu P. Relationship of elevated neural cell adhesion molecule 1 with interleukin-10 and disease severity in bipolar disorder. Asian J Psychiatr. 2019;47:101849.

12. Wu Y, Li H, Zhou Y, Yu J, Zhang Y, Song M, et al. Sex-specific neural circuits of emotion regulation in the centromedial amygdala. Sci Rep. 2016;6:23112.

13. Roy AK, Shehzad Z, Margulies DS, Kelly AM, Uddin LQ, Gotimer K, et al. Functional connectivity of the human amygdala using resting state fMRI. Neuroimage. 2009;45(2):614-26.

14. Jalbrzikowski M, Larsen B, Hallquist MN, Foran W, Calabro F, Luna B. Development of White Matter Microstructure and Intrinsic Functional Connectivity Between the Amygdala and Ventromedial Prefrontal Cortex: Associations with Anxiety and Depression. Biol Psychiatry. 2017;82(7):511-21.

15. Mosher CP, Zimmerman PE, Gothard KM. Response characteristics of basolateral and centromedial neurons in the primate amygdala. J Neurosci. 2010;30(48):16197-207.

16. Pape HC, Pare D. Plastic synaptic networks of the amygdala for the acquisition, expression, and extinction of conditioned fear. Physiol Rev. 2010;90(2):419-63.

17. Bzdok D, Laird AR, Zilles K, Fox PT, Eickhoff SB. An investigation of the structural, connectional, and functional subspecialization in the human amygdala. Hum Brain Mapp. 2013;34(12):3247-66.

18. Janak PH, Tye KM. From circuits to behaviour in the amygdala. Nature. 2015;517(7534):284-92.

19. Zhang L, Opmeer EM, van der Meer L, Aleman A, Curcic-Blake B, Ruhe HG. Altered frontal-amygdala effective connectivity during effortful emotion regulation in bipolar disorder. Bipolar Disord. 2018;20(4):349-58.

20. Tang $Y, M a Y$, Chen X, Fan X, Jiang X, Zhou Y, et al. Age-specific effects of structural and functional connectivity in prefrontal-amygdala circuitry in women with bipolar disorder. BMC Psychiatry. 2018;18(1):177.

21. Ho NF, Li HCP, Lee DR, Chew QH, Chen G, Sim K. The Amygdala in Schizophrenia and Bipolar Disorder: A Synthesis of Structural MRI, Diffusion Tensor Imaging, and Resting-State Functional 
Connectivity Findings. Harv Rev Psychiatry. 2019;27(3):150-64.

22. Li G, Liu P, Andari E, Zhang A, Zhang K. The Role of Amygdala in Patients with Euthymic Bipolar Disorder During Resting State. Front Psychiatry. 2018;9:445.

23. Ambrosi E, Arciniegas DB, Madan A, Curtis KN, Patriquin MA, Jorge RE, et al. Insula and amygdala resting-state functional connectivity differentiate bipolar from unipolar depression. Acta Psychiatr Scand. 2017;136(1):129-39.

24. Dinarello CA. Proinflammatory cytokines. Chest. 2000;118(2):503-8.

25. Capuron L, Miller AH. Immune system to brain signaling: neuropsychopharmacological implications. Pharmacol Ther. 2011;130(2):226-38.

26. Rosenblat JD, Mclntyre RS. Bipolar Disorder and Inflammation. Psychiatr Clin North Am. 2016;39(1):125-37.

27. Najjar S, Pearlman DM, Alper K, Najjar A, Devinsky O. Neuroinflammation and psychiatric illness. J Neuroinflammation. 2013;10:43.

28. Pan AY, Ryu E, Geske JR, Zhou XY, McElroy SL, Cicek MS, et al. The impact of sample processing on inflammatory markers in serum: Lessons learned. World J Biol Psychiatry. 2020;21(3):230-7.

29. Modabbernia A, Taslimi S, Brietzke E, Ashrafi M. Cytokine alterations in bipolar disorder: a metaanalysis of 30 studies. Biol Psychiatry. 2013;74(1):15-25.

30. Brietzke E, Stertz L, Fernandes BS, Kauer-Sant'Anna M, Mascarenhas M, Escosteguy VA, et al. Comparison of cytokine levels in depressed, manic and euthymic patients with bipolar disorder. $J$ Affect Disord. 2009;116(3):214-7.

31. Isgren A, Sellgren C, Ekman CJ, Holmen-Larsson J, Blennow K, Zetterberg H, et al. Markers of neuroinflammation and neuronal injury in bipolar disorder: Relation to prospective clinical outcomes. Brain Behav Immun. 2017;65:195-201.

32. Fiedorowicz JG, Prossin AR, Johnson CP, Christensen GE, Magnotta VA, Wemmie JA. Peripheral inflammation during abnormal mood states in bipolar I disorder. J Affect Disord. 2015;187:172-8.

33. Rowland T, Perry BI, Upthegrove R, Barnes N, Chatterjee J, Gallacher D, et al. Neurotrophins, cytokines, oxidative stress mediators and mood state in bipolar disorder: systematic review and meta-analyses. Br J Psychiatry. 2018;213(3):514-25.

34. Chen MH, Chang WC, Hsu JW, Huang KL, Tu PC, Su TP, et al. Correlation of proinflammatory cytokines levels and reduced gray matter volumes between patients with bipolar disorder and unipolar depression. J Affect Disord. 2019;245:8-15.

35. Wilczynska K, Simonienko K, Konarzewska B, Szajda SD, Waszkiewicz N. Morphological changes of the brain in mood disorders. Psychiatr Pol. 2018;52(5):797-805.

36. Felger JC. Imaging the Role of Inflammation in Mood and Anxiety-related Disorders. Curr Neuropharmacol. 2018;16(5):533-58.

37. Tu PC, Li CT, Lin WC, Chen MH, Su TP, Bai YM. Structural and functional correlates of serum soluble IL-6 receptor level in patients with bipolar disorder. J Affect Disord. 2017;219:172-7. 
38. Yan CG, Wang XD, Zuo XN, Zang YF. DPABI: Data Processing \&amp; Analysis for (Resting-State) Brain Imaging. Neuroinformatics. 2016;14(3):339-51.

39. Jenkinson M, Bannister P, Brady M, Smith S. Improved optimization for the robust and accurate linear registration and motion correction of brain images. Neuroimage. 2002;17(2):825-41.

40. Yan CG, Cheung B, Kelly C, Colcombe S, Craddock RC, Di Martino A, et al. A comprehensive assessment of regional variation in the impact of head micromovements on functional connectomics. Neuroimage. 2013;76:183-201.

41. Fox MD, Zhang D, Snyder AZ, Raichle ME. The global signal and observed anticorrelated resting state brain networks. J Neurophysiol. 2009;101(6):3270-83.

42. Yan CG, Craddock RC, Zuo XN, Zang YF, Milham MP. Standardizing the intrinsic brain: towards robust measurement of inter-individual variation in 1000 functional connectomes. Neuroimage. 2013;80:246-62.

43. Van Dijk KR, Sabuncu MR, Buckner RL. The influence of head motion on intrinsic functional connectivity MRI. Neuroimage. 2012;59(1):431-8.

44. Friston KJ, Williams S, Howard R, Frackowiak RS, Turner R. Movement-related effects in fMRI timeseries. Magn Reson Med. 1996;35(3):346-55.

45. Fan L, Li H, Zhuo J, Zhang Y, Wang J, Chen L, et al. The Human Brainnetome Atlas: A New Brain Atlas Based on Connectional Architecture. Cereb Cortex. 2016;26(8):3508-26.

46. Ochsner KN, Ray RR, Hughes B, McRae K, Cooper JC, Weber J, et al. Bottom-up and top-down processes in emotion generation: common and distinct neural mechanisms. Psychol Sci. 2009;20(11):1322-31.

47. Mukherjee P, Sabharwal A, Kotov R, Szekely A, Parsey R, Barch DM, et al. Disconnection Between Amygdala and Medial Prefrontal Cortex in Psychotic Disorders. Schizophr Bull. 2016;42(4):1056-67.

48. Chen G, Zhao L, Jia Y, Zhong S, Chen F, Luo X, et al. Abnormal cerebellum-DMN regions connectivity in unmedicated bipolar II disorder. J Affect Disord. 2019;243:441-7.

49. Zhong Y, Wang C, Gao W, Xiao Q, Lu D, Jiao Q, et al. Aberrant Resting-State Functional Connectivity in the Default Mode Network in Pediatric Bipolar Disorder Patients with and without Psychotic Symptoms. Neurosci Bull. 2019;35(4):581-90.

50. Wang Y, Zhong S, Jia Y, Sun Y, Wang B, Liu T, et al. Disrupted Resting-State Functional Connectivity in Nonmedicated Bipolar Disorder. Radiology. 2016;280(2):529-36.

51. Wang Y, Zhong S, Jia Y, Zhou Z, Zhou Q, Huang L. Reduced interhemispheric resting-state functional connectivity in unmedicated bipolar II disorder. Acta Psychiatr Scand. 2015;132(5):400-7.

52. Yu HL, Liu WB, Wang T, Huang PY, Jie LY, Sun JZ, et al. Difference in resting-state fractional amplitude of low-frequency fluctuation between bipolar depression and unipolar depression patients. Eur Rev Med Pharmacol Sci. 2017;21(7):1541-50.

53. Tsujii N, Mikawa W, Adachi T, Hirose T, Shirakawa O. Shared and differential cortical functional abnormalities associated with inhibitory control in patients with schizophrenia and bipolar disorder. 
Sci Rep. 2018;8(1):4686.

54. Tseng WL, Thomas LA, Harkins E, Stoddard J, Zarate CJ, Pine DS, et al. Functional connectivity during masked and unmasked face emotion processing in bipolar disorder. Psychiatry Res Neuroimaging. 2016;258:1-9.

55. Vai B, Poletti S, Radaelli D, Dallaspezia S, Bulgarelli C, Locatelli C, et al. Successful antidepressant chronotherapeutics enhance fronto-limbic neural responses and connectivity in bipolar depression. Psychiatry Res. 2015;233(2):243-53.

56. Savitz JB, Price JL, Drevets WC. Neuropathological and neuromorphometric abnormalities in bipolar disorder: view from the medial prefrontal cortical network. Neurosci Biobehav Rev. 2014;42:132-47.

57. Rajkowska G, Halaris A, Selemon LD. Reductions in neuronal and glial density characterize the dorsolateral prefrontal cortex in bipolar disorder. Biol Psychiatry. 2001;49(9):741-52.

58. Olson IR, Plotzker A, Ezzyat Y. The Enigmatic temporal pole: a review of findings on social and emotional processing. Brain. 2007;130(Pt 7):1718-31.

59. Vai B, Serretti A, Poletti S, Mascia M, Lorenzi C, Colombo C, et al. Cortico-limbic functional connectivity mediates the effect of early life stress on suicidality in bipolar depressed $5-H T T L P R * S$ carriers. J Affect Disord. 2020;263:420-7.

60. Yin Z, Chang M, Wei S, Jiang X, Zhou Y, Cui L, et al. Decreased Functional Connectivity in Insular Subregions in Depressive Episodes of Bipolar Disorder and Major Depressive Disorder. Front Neurosci. 2018;12:842.

61. Zhang L, Wu H, Xu J, Shang J. Abnormal Global Functional Connectivity Patterns in Medication-Free Major Depressive Disorder. Front Neurosci. 2018;12:692.

62. Borras-Ferris L, Perez-Ramirez U, Moratal D. Link-Level Functional Connectivity Neuroalterations in Autism Spectrum Disorder: A Developmental Resting-State fMRI Study. Diagnostics (Basel). 2019;9(1).

63. Zuliani R, Moorhead TW, Job D, McKirdy J, Sussmann JE, Johnstone EC, et al. Genetic variation in the G72 (DAOA) gene affects temporal lobe and amygdala structure in subjects affected by bipolar disorder. Bipolar Disord. 2009;11(6):621-7.

64. Neves MC, Albuquerque MR, Malloy-Diniz L, Nicolato R, Silva NF, de Souza-Duran FL, et al. A voxelbased morphometry study of gray matter correlates of facial emotion recognition in bipolar disorder. Psychiatry Res. 2015;233(2):158-64.

65. Kasai K, Shenton ME, Salisbury DF, Onitsuka T, Toner SK, Yurgelun-Todd D, et al. Differences and similarities in insular and temporal pole MRI gray matter volume abnormalities in first-episode schizophrenia and affective psychosis. Arch Gen Psychiatry. 2003;60(11):1069-77.

66. Spuhler K, Bartlett E, Ding J, DeLorenzo C, Parsey R, Huang C. Diffusion Entropy: A Potential Neuroimaging Biomarker of Bipolar Disorder in the Temporal Pole. Synapse. 2018;72(2).

67. Zhou Q, Womer FY, Kong L, Wu F, Jiang X, Zhou Y, et al. Trait-Related Cortical-Subcortical Dissociation in Bipolar Disorder: Analysis of Network Degree Centrality. J Clin Psychiatry. 2017;78(5):584-91. 
68. Van der Schot A, Kahn R, Ramsey N, Nolen W, Vink M. Trait and state dependent functional impairments in bipolar disorder. Psychiatry Res. 2010;184(3):135-42.

69. Liu TY, Chen YS, Su TP, Hsieh JC, Chen LF. Abnormal early gamma responses to emotional faces differentiate unipolar from bipolar disorder patients. Biomed Res Int. 2014;2014:906104.

70. Pantovic-Stefanovic M, Petronijevic N, Dunjic-Kostic B, Velimirovic M, Nikolic T, Jurisic V, et al. sVCAM-1, sICAM-1, TNF-alpha and IL-6 levels in bipolar disorder type I: Acute, longitudinal and therapeutic implications. World J Biol Psychiatry. 2018;19(sup2):S41-51.

71. Haapakoski R, Mathieu J, Ebmeier KP, Alenius H, Kivimaki M. Cumulative meta-analysis of interleukins 6 and 1beta, tumour necrosis factor alpha and C-reactive protein in patients with major depressive disorder. Brain Behav Immun. 2015;49:206-15.

72. Niu Z, Yang L, Wu X, Zhu Y, Chen J, Fang Y. The Relationship Between Neuroimmunity and Bipolar Disorder: Mechanism and Translational Application. Neurosci Bull. 2019;35(4):595-607.

73. Sayana P, Colpo GD, Simoes LR, Giridharan VV, Teixeira AL, Quevedo J, et al. A systematic review of evidence for the role of inflammatory biomarkers in bipolar patients. J Psychiatr Res. 2017;92:16082.

74. Karabulut S, Tasdemir I, Akcan U, Kucukali Cl, Tuzun E, Cakir S. [Inflammation and Neurodegeneration in Patients with Early-Stageand Chronic Bipolar Disorder]. Turk Psikiyatri Derg. 2019;30(2):75-81.

75. Fan C, Song Q, Wang P, Li Y, Yang M, Liu B, et al. Curcumin Protects Against Chronic Stress-induced Dysregulation of Neuroplasticity and Depression-like Behaviors via Suppressing IL-1beta Pathway in Rats. Neuroscience. 2018;392:92-106.

76. Davies KA, Cooper E, Voon V, Tibble J, Cercignani M, Harrison NA. Interferon and anti-TNF therapies differentially modulate amygdala reactivity which predicts associated bidirectional changes in depressive symptoms. Mol Psychiatry. 2020.

77. Mehta ND, Haroon E, Xu X, Woolwine BJ, Li Z, Felger JC. Inflammation negatively correlates with amygdala-ventromedial prefrontal functional connectivity in association with anxiety in patients with depression: Preliminary results. Brain Behav Immun. 2018;73:725-30.

78. Muscatell KA, Dedovic K, Slavich GM, Jarcho MR, Breen EC, Bower JE, et al. Greater amygdala activity and dorsomedial prefrontal-amygdala coupling are associated with enhanced inflammatory responses to stress. Brain Behav Immun. 2015;43:46-53.

79. Liu K, Zhao X, Lu X, Zhu X, Chen H, Wang M, et al. Effect of selective serotonin reuptake inhibitor on prefrontal-striatal connectivity is dependent on the level of TNF-alpha in patients with major depressive disorder. Psychol Med. 2019;49(15):2608-16.

80. Mclntosh RC, Paul R, Ndhlovu LC, Hidalgo M, Lobo JD, Walker M, et al. Resting-state connectivity and spontaneous activity of ventromedial prefrontal cortex predict depressive symptomology and peripheral inflammation in HIV. J Neurovirol. 2018;24(5):616-28.

\section{Figures}



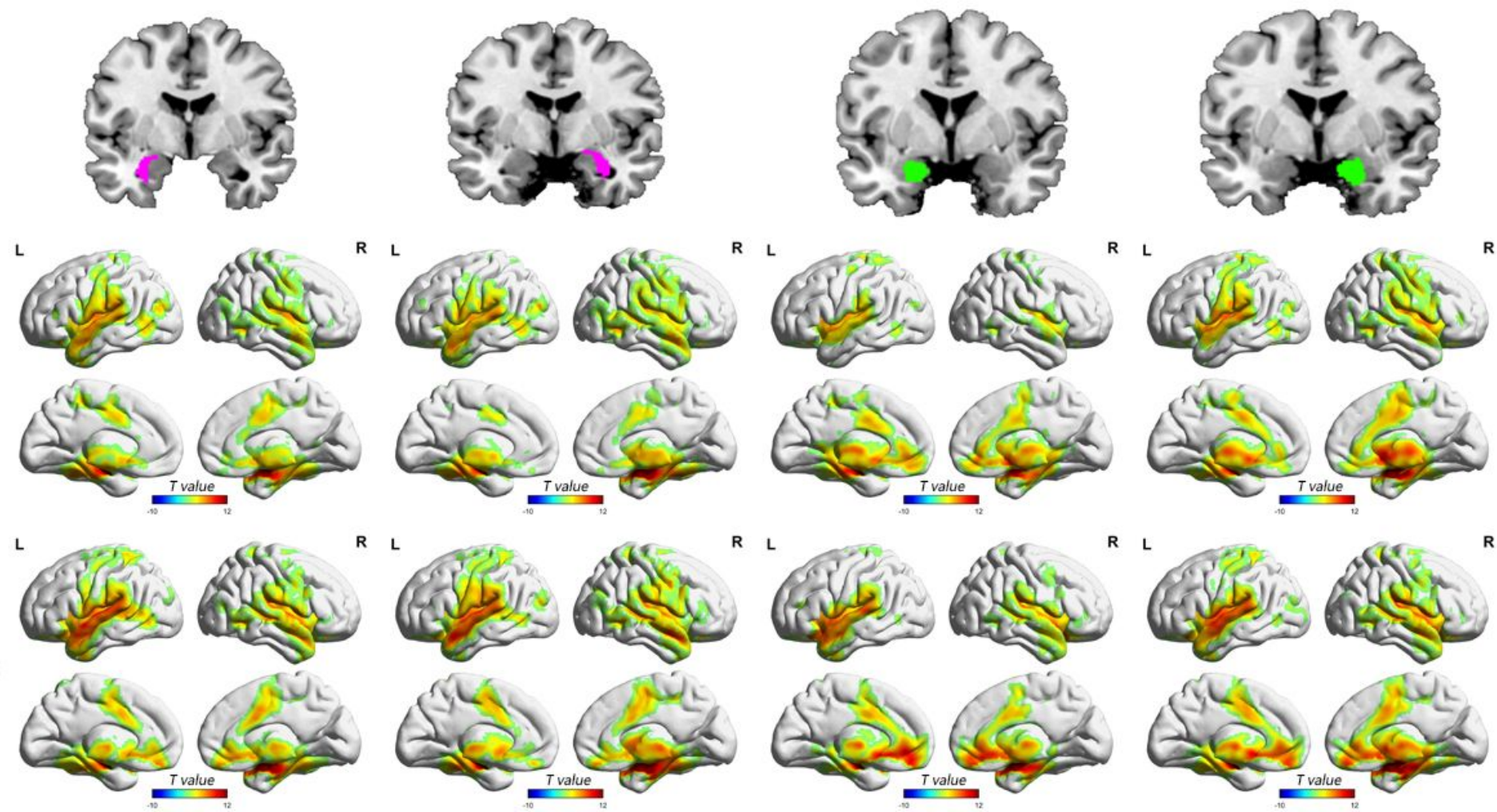

\section{Figure 1}

The FC patterns of the lateral amygdala, medial amygdala in the BD patients and $\mathrm{HCs}(\mathrm{p}<0.05$, uncorrected). The color bar represents functional connection. The $T$ value indicated the intensity of the significant difference which was obtained by one-sample t-test. FC, functional connectivity; BD, bipolar disorder; $\mathrm{HCs}$, healthy controls. 
Seed: L medial amygdala

.32
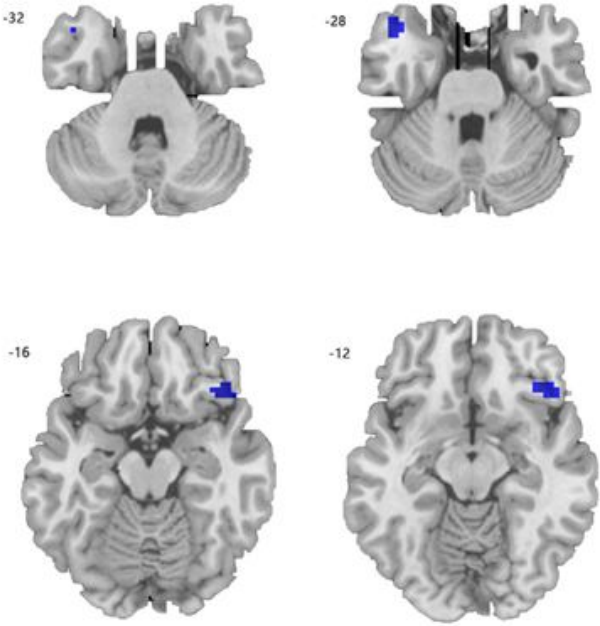

$T$ value
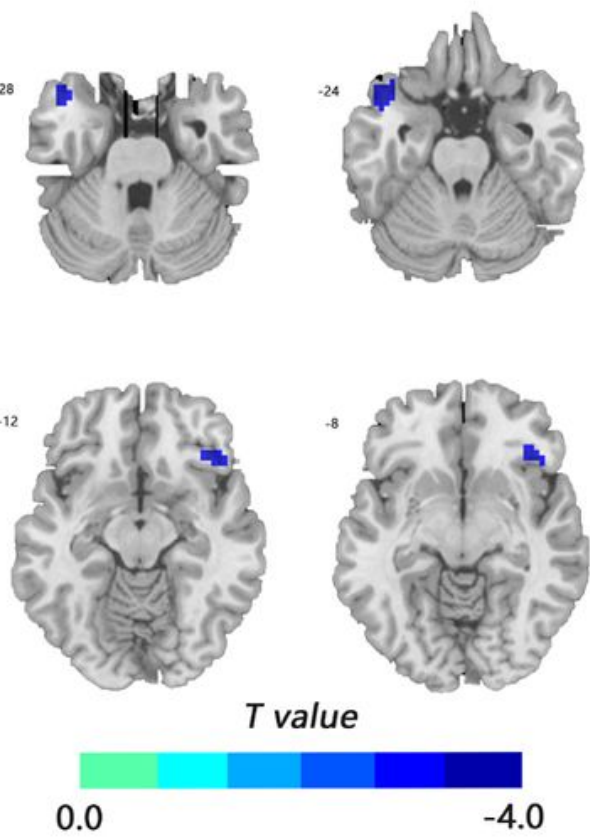

Seed: $\mathrm{R}$ medial amygdala
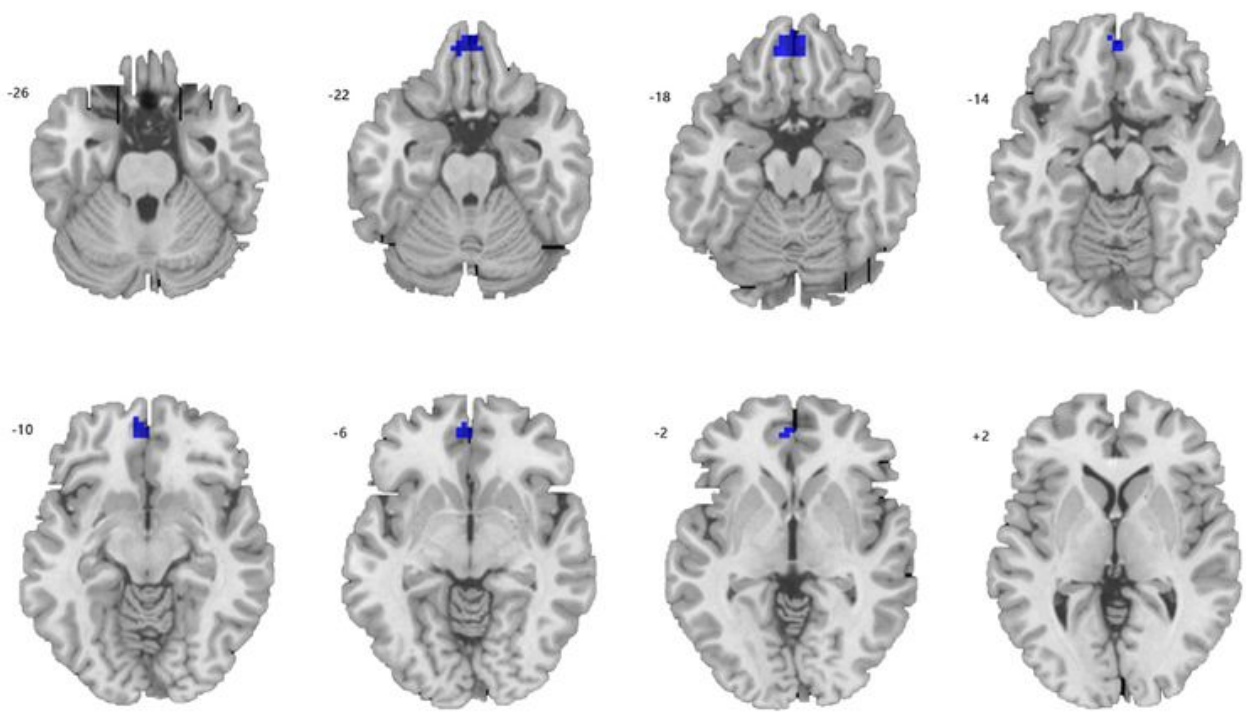

$T$ value

L

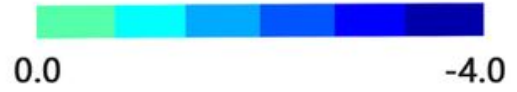

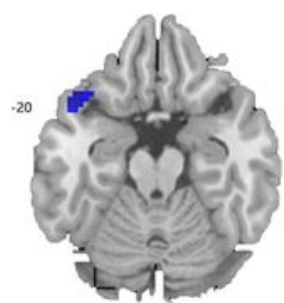

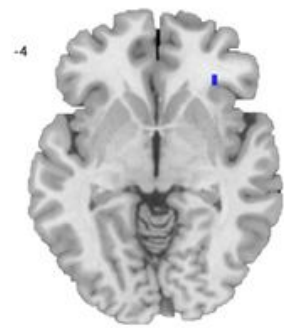



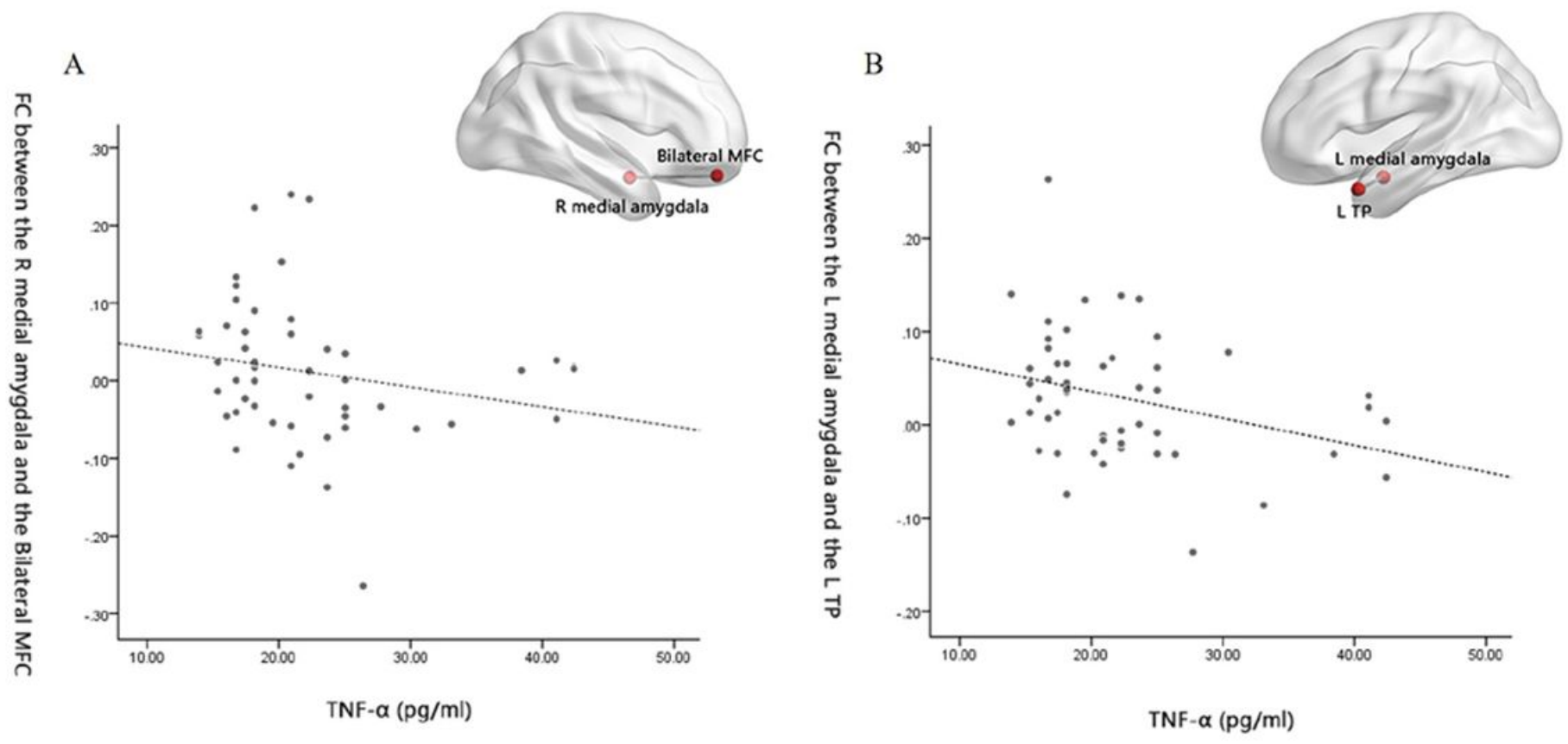

Figure 3

The correlation between abnormal FC values ( $A$ : R medial amygdala-bilateral MFC, $r=-0.320, p=0.022$; $B$ : $L$ medial amygdala- $L T P, r=-0.320, p=0.021)$ and TNF- $a(p<0.05)$. FC, functional connectivity; $L(R)$, left (right) hemisphere; TP, temporal pole; MFC, medial frontal cortex; TNF, tumor necrosis factor.

\section{Supplementary Files}

This is a list of supplementary files associated with this preprint. Click to download.

- Supplementarymaterials.docx 